HUB-EP-01/18

\title{
Comments on the Energy-Momentum Tensor in Non-Commutative Field Theories
}

\author{
Mohab Abou-Zeid[ and Harald Dorn] \\ Institut für Physik, Humboldt Universität zu Berlin, Invalidenstrasse 110, \\ D-10115 Berlin, Germany
}

\begin{abstract}
In a non-commutative field theory, the energy-momentum tensor obtained from the Noether method needs not be symmetric; in a massless theory, it needs not be traceless either. In a non-commutative scalar field theory, the method yields a locally conserved yet non-symmetric energy-momentum tensor whose trace does not vanish for massless fields. A non-symmetric tensor also governs the response of the action to a general coordinate transformation. In non-commutative gauge theory, if translations are suitably combined with gauge transformations, the method yields a covariantly constant tensor which is symmetric but only gauge covariant. Using suitable Wilson functionals, this can be improved to yield a locally conserved and gauge invariant, albeit non-symmetric, energy-momentum tensor.
\end{abstract}

April, 2001

\footnotetext{
${ }^{1}$ abouzeid@physik.hu-berlin.de

22dorn@physik.hu-berlin.de
} 


\section{Introduction}

Ordinary field theory on a flat non-commutative space-time in which the coordinates satisfy the commutation relations

$$
\left[x^{\mu}, x^{\nu}\right]=i \theta^{\mu \nu}
$$

(where $\theta^{\mu \nu}$ is a constant real antisymmetric matrix) can alternatively be described as field theory on an ordinary space-time but with a Moyal deformed (non-commutative) product structure. Many classical, perturbative and non-perturbative aspects of non-commutative field theories have been studied (see e.g. [1, 2, 3, 4, 5, 6] and references therein). They also arise in a certain limit of string theory (see e.g. [7, 8, 9]).

In this note we comment on the definition and properties of the energy-momentum tensor in such theories. This issue has previously been discussed for scalar $\phi^{4}$-theory in [10, 11, 12]. For D-branes in a constant background $B$ field in bosonic and superstring theory, the symmetric energy-momentum tensors were constructed in [13, 14].

\section{Non-Commutative Scalar Field Theory}

Consider first the non-commutative real scalar field theory in a flat four dimensional space-time of Minkowski signature described by the action

$$
S=\int d x\left(\frac{1}{2} \partial_{\mu} \phi \star \partial^{\mu} \phi-\frac{m^{2}}{2} \phi \star \phi-\frac{\lambda}{4 !} \phi \star \phi \star \phi \star \phi\right),
$$

where $\star$ denotes the standard Moyal $\star$-product. In the following, we will make repeated use of the cyclic property of the integration over space-time. We will also use the products $\star^{\prime}$ (introduced in [15, 16]) and $\star^{\prime \prime}$ (introduced in [11]) defined by

$$
\left.\left(f \star^{\prime} g\right)(x) \equiv \frac{\sin \left(\frac{1}{2} \frac{\partial}{\partial x_{\mu}} \theta^{\mu \nu} \frac{\partial}{\partial y_{\nu}}\right)}{\frac{1}{2} \frac{\partial}{\partial x_{\mu}} \theta^{\mu \nu} \frac{\partial}{\partial y_{\nu}}} f(x) g(y)\right|_{y=x}
$$

and

$$
\left.\left(f \star^{\prime \prime} g\right)(x) \equiv \frac{\cos \left(\frac{1}{2} \frac{\partial}{\partial x_{\mu}} \theta^{\mu \nu} \frac{\partial}{\partial y_{\nu}}\right)-1}{\frac{1}{2} \frac{\partial}{\partial x_{\mu}} \theta^{\mu \nu} \frac{\partial}{\partial y_{\nu}}} f(x) g(y)\right|_{y=x} .
$$

All three products $\star, \star^{\prime}$ and $\star^{\prime \prime}$ satisfy the Leibnitz rule. Moreover, $\star$ is associative and non-commutative, $\star^{\prime}$ is non-associative and commutative, and $\star^{\prime \prime}$ is nonassociative and anti-commutative. Furthermore, they are related by the formulas

$$
\theta^{\mu \nu} \partial_{\mu} f \star^{\prime} \partial_{\nu} g=-i[f, g]_{\star}
$$


and

$$
\theta^{\mu \nu} \partial_{\mu} f \star^{\prime \prime} \partial_{\nu} g=\{f, g\}_{\star}-2 f g
$$

Here we defined the Moyal brackets

$$
[f, g]_{\star} \equiv f \star g-g \star f, \quad\{f, g\}_{\star} \equiv f \star g+g \star f .
$$

The field equation which follows from action (2) is

$$
\partial^{2} \phi+m^{2} \phi+\frac{\lambda}{3 !} \phi \star \phi \star \phi=0
$$

Applying the Noether method to the action (2) one finds the equation [10, 11, 12,

$$
\partial_{\mu} T^{\mu \nu}=\frac{\lambda}{4 !}\left[\left[\phi, \partial^{\nu} \phi\right]_{\star}, \phi \star \phi\right]_{\star},
$$

where

$$
T_{\mu \nu}=\frac{1}{2}\left\{\partial_{\mu} \phi, \partial_{\nu} \phi\right\}_{\star}-\eta_{\mu \nu}\left(\frac{1}{2} \partial_{\rho} \phi \star \partial^{\rho} \phi-\frac{m^{2}}{2} \phi \star \phi-\frac{\lambda}{4 !} \phi \star \phi \star \phi \star \phi\right) .
$$

If one chooses $T_{\mu \nu}$ as the energy-momentum tensor, then energy-momentum is not conserved locally. On the other hand, due to the fact that integrals over $\star-$ commutators vanish, one still finds global conservation of energy and momentum [10, 12], at least for theories without time-space non-commutativity.

Using (5) twice, together with the antisymmetry of $\theta^{\mu \nu}$, the r.h.s. of (9) can be written as $-\partial_{\mu} t^{\mu \nu}$ with $t^{\mu \nu}$ defined by

$$
t^{\mu \nu} \equiv \frac{\lambda}{4 !} \theta^{\mu \rho} \theta^{\alpha \beta}\left[a\left(\partial_{\alpha} \phi \star^{\prime} \partial_{\beta} \partial^{\nu} \phi\right) \star^{\prime} \partial_{\rho}(\phi \star \phi)-(1-a) \partial_{\rho}\left(\partial_{\alpha} \phi \star^{\prime} \partial_{\beta} \partial^{\nu} \phi\right) \star^{\prime}(\phi \star \phi)\right],
$$

where $a$ is a free (real) parameter. This implies that the energy-momentum tensor

$$
\mathbf{T}^{\mu \nu} \equiv T^{\mu \nu}+t^{\mu \nu}
$$

is locally conserved!'

$$
\partial_{\mu} \mathbf{T}^{\mu \nu}=0
$$

For $m=0$, the trace of the symmetric part of (12) can be cancelled by an additional term which does not contribute to its divergence. The 'improved' tensor [17]

$$
\mathbf{T}_{\mu \nu}^{I} \equiv \mathbf{T}_{\mu \nu}^{m=0}+\frac{1}{6}\left(\eta_{\mu \nu} \partial^{2}-\partial_{\mu} \partial_{\nu}\right) \phi \star \phi
$$

\footnotetext{
${ }^{3}$ The case $a=0$ has been considered in 11 .
} 
has vanishing divergence, however its trace does not vanish; instead it is given by

$$
t_{\mu}^{\mu}=\frac{\lambda}{4 !} \theta^{\mu \rho} \theta^{\alpha \beta}\left[a\left(\partial_{\alpha} \phi \star^{\prime} \partial_{\beta} \partial_{\mu} \phi\right) \star^{\prime} \partial_{\rho}(\phi \star \phi)-(1-a) \partial_{\rho}\left(\partial_{\alpha} \phi \star^{\prime} \partial_{\beta} \partial_{\mu} \phi\right) \star^{\prime}(\phi \star \phi)\right] .
$$

This curious nonvanishing trace in a massless theory is allowed because the theory is not scale invariant (recall that the non-commutativity parameter $\theta$ has dimensions of length squared)国.

The energy-momentum tensor $\mathbf{T}_{\mu \nu}$ is locally conserved, but it is not symmetric because of the non-symmetric contribution $t_{\mu \nu}$. Although this may appear unphysical at first sight, a closer look shows that the usual arguments for symmetry do not apply here. In ordinary field theory, the symmetry of the energy-momentum tensor is a consequence of the requirements that the angular momentum density is locally conserved and expressible by the familiar formula

$$
M^{\mu \nu \rho}=x^{\mu} \mathbf{T}^{\nu \rho}-x^{\nu} \mathbf{T}^{\mu \rho}
$$

In the present case, however, the theory is not Lorentz invariant. In particular, the non-commutativity parameter $\theta^{\mu \nu}$ generically breaks the rotational part of the Lorentz group, so that the angular momentum density is not conserved. If (16) is imposed, then the energy-momentum tensor is necessarily non-symmetric. On the other hand, if (16) is abandoned, no conclusion can be drawn concerning the symmetry of the energy-momentum tensorf.

\subsection{The Response to Diffeomorphisms}

Another argument leading to the same conclusion is as follows. Usually, the symmetry of the energy-momentum tensor comes from the way the theory has to couple to gravity. We do not know how this is realized in our non-commutative field theory, but we can investigate the related issue of the response of the flat space version to general coordinate transformations. Consider the variation of a Lagrangian density $L[\phi]$ under an infinitesimal coordinate transformation $x^{\prime}=x+\epsilon(x)$. Assuming that $\phi$ is a coordinate scalar, its variation is $\delta \phi=-\mathcal{L}_{\epsilon} \phi=-\epsilon^{\mu} \partial_{\mu} \phi$ and moreover

$$
L\left[\phi^{\prime}\left(x^{\prime}\right)\right]-L[\phi(x)]=\epsilon^{\mu} \partial_{\mu} L+L[\phi(x)+\delta \phi(x)]-L[\phi(x)]+O\left(\epsilon^{2}\right) .
$$

\footnotetext{
${ }^{4}$ By contrast, the authors of [12] attribute the violation of scale invariance to the local nonconservation of the symmetric tensor $T^{\mu \nu}$.

${ }^{5}$ In ref. [1], the energy-momentum tensor is non-symmetric and formula (16) is modified.
} 
For the non-commutative scalar theory with action (2), we assume for the sake of simplicity that the Moyal product $\star$ always refers to the original coordinates $x$ (in other words, $\theta^{\mu \nu}$ is treated as a coordinate tensor). This leads to

$$
\begin{aligned}
L\left[\phi^{\prime}\left(x^{\prime}\right)\right]-L[\phi(x)]= & -\epsilon^{\nu} \partial^{\mu} T_{\mu \nu}+\frac{\lambda}{4 !}\left[\left[\phi, \epsilon^{\nu} \partial_{\nu} \phi\right]_{\star}, \phi \star \phi\right]_{\star}-\frac{1}{2}\left\{\frac{\delta S}{\delta \phi}, \epsilon^{\nu} \partial_{\nu} \phi\right\}_{\star} \\
& +\frac{1}{2} \epsilon^{\nu} \partial^{\mu}\left\{\partial_{\mu} \phi, \partial_{\nu} \phi\right\}_{\star}-\frac{1}{2} \partial^{\mu}\left\{\partial_{\mu} \phi, \epsilon^{\nu} \partial_{\nu} \phi\right\}_{\star}+O\left(\epsilon^{2}\right) .
\end{aligned}
$$

For constant $\epsilon$, due to translational invariance, the l.h.s. is zero, the fourth and fifth terms on the r.h.s. cancel and $\epsilon^{\nu}$ in the second and third terms can be taken out of the $\star$-commutator. On shell, this implies eq. (9) and its reformulation (13), respectively. Off shell, this implies the identity

$$
-\partial^{\mu} T_{\mu \nu}+\frac{\lambda}{4 !}\left[\left[\phi, \partial_{\nu} \phi\right]_{\star}, \phi \star \phi\right]_{\star}-\frac{1}{2}\left\{\frac{\delta S}{\delta \phi}, \partial_{\nu} \phi\right\}_{\star}=0 .
$$

On the other hand, we may consider the response of the action under a general coordinate transformation. Using (19), one finds up to $O\left(\epsilon^{2}\right)$ :

$$
\begin{aligned}
L\left[\phi^{\prime}\left(x^{\prime}\right)\right]-L[\phi(x)]= & \frac{1}{2} \epsilon^{\nu} \partial^{\mu}\left\{\partial_{\mu} \phi, \partial_{\nu} \phi\right\}_{\star}-\frac{1}{2} \partial^{\mu}\left\{\partial_{\mu} \phi, \epsilon^{\nu} \partial_{\nu} \phi\right\}_{\star} \\
& +\frac{\lambda}{4 !}\left(\left[\left[\phi, \epsilon^{\nu} \partial_{\nu} \phi\right]_{\star}, \phi \star \phi\right]_{\star}-\epsilon^{\nu}\left[\left[\phi, \partial_{\nu} \phi\right]_{\star}, \phi \star \phi\right]_{\star}\right) \\
& -\frac{1}{2}\left(\left\{\frac{\delta S}{\delta \phi}, \epsilon^{\nu} \partial_{\nu} \phi\right\}_{\star}-\epsilon^{\nu}\left\{\frac{\delta S}{\delta \phi}, \partial_{\nu} \phi\right\}_{\star}\right) .
\end{aligned}
$$

Note that under the space-time integral, total derivatives and Moyal commutators will not contribute; moreover, the Moyal product in the penultimate term can be replaced with ordinary multiplication. Utilizing (6), we find

$$
S\left[\phi^{\prime}\left(x^{\prime}\right)\right]-S[\phi(x)]=-\int d x\left(\mathbf{T}^{\mu \nu}+\frac{1}{2} \theta^{\mu \rho} \frac{\delta S}{\delta \phi} \star^{\prime \prime} \partial_{\rho} \partial^{\nu} \phi\right) \partial_{\mu} \epsilon_{\nu}+O\left(\epsilon^{2}\right) .
$$

This result is valid for arbitrary field configurations and arbitrary diffeomorphisms $\epsilon$. Thus the response to general coordinate transformations is governed by the non-symmetric but locally conserved tensor $\mathbf{T}^{\mu \nu}+\frac{1}{2} \theta^{\mu \rho} \frac{\delta S}{\delta \phi} \star^{\prime \prime} \partial_{\rho} \partial^{\nu} \phi$. Although the non-symmetry is consistent with our previous argument based on broken Lorentz invariance, it is not clear to us whether this result has any bearing on the existence of a diffeomorphism-invariant generalization of the theory (2). 


\subsection{The Belinfante Problem}

Although we have just argued that the usual physical reasons for the symmetry of the energy-momentum tensor do not apply, one nevertheless may wonder whether a further contribution can be added to (12) in order to make it symmetric, along the lines of the Belinfante procedure [18]. Consider adding to $\mathbf{T}_{\mu \nu}$ a contribution of the form $\partial^{\rho} \chi_{\rho \mu \nu}$, where the tensor $\chi_{\rho \mu \nu}$ is antisymmetric in its first two indices,

$$
\chi_{\rho \mu \nu}=\chi_{[\rho \mu] \nu}
$$

Such a contribution would clearly not change the divergence of $T_{\mu \nu}$. Thus the tensor

$$
\hat{\mathbf{T}}_{\mu \nu} \equiv \mathbf{T}_{\mu \nu}+\partial^{\rho} \chi_{\rho \mu \nu}
$$

would also be locally conserved, and it would be symmetric provided $\chi_{\rho \mu \nu}$ is a solution to the differential equation

$$
\begin{aligned}
2 \partial^{\rho} \chi_{\rho}^{[\mu \nu]}=-\frac{\lambda}{4 !} & {\left[\theta ^ { \mu \rho } \theta ^ { \alpha \beta } \left(a\left(\partial_{\alpha} \phi \star^{\prime} \partial_{\beta} \partial^{\nu} \phi\right) \star^{\prime} \partial_{\rho}(\phi \star \phi)\right.\right.} \\
& \left.\left.-(1-a) \partial_{\rho}\left(\partial_{\alpha} \phi \star^{\prime} \partial_{\beta} \partial^{\nu} \phi\right) \star^{\prime}(\phi \star \phi)\right)-(\mu \leftrightarrow \nu)\right] .
\end{aligned}
$$

In the case $m=0$, the additional condition of vanishing trace would require that $\chi_{\rho} \equiv \eta^{\mu \nu} \chi_{\rho \mu \nu}$ satisfies

$$
\partial^{\rho} \chi_{\rho}=-t_{\mu}^{\mu}
$$

There is no violated integrability condition for these two differential equations, which certainly admit integral solutions. The problem consists in finding a solution which depends on $x$ only via the dependence on the field $\phi$ and its derivatives. There is no solution within an ansatz taking into account the lowest orders in the number of derivatives and the relevant $\star$ and $\star^{\prime}$ powers of $\phi$. Since there are mixings between different orders, we cannot exclude the existence of suitable solutions, but on the basis of a cursory search we suspect the absence of any solution of the required type.

\section{Non-Commutative Gauge Theory}

We now turn to the case of non-commutative $U(N)$ Yang-Mills theory in $D$ dimensions, with action

$$
S=\int d x \operatorname{tr} F_{\mu \nu} \star F^{\mu \nu}
$$

where $F_{\mu \nu}$ denotes the strength of the non-commutative Yang-Mills gauge field $A_{\mu}$. In standard Yang-Mills theory the canonical energy-momentum tensor is neither 
symmetric nor gauge invariant. By applying the Belinfante procedure, one finds a symmetric and gauge invariant improved tensor. However, there is a more direct way to get the improved tensor by combining in the Noether procedure translations with suitably adapted gauge transformations [19]. Applying this method to noncommutative Yang-Mills theory we get

$$
D_{\mu} T^{\mu \nu} \equiv \partial_{\mu} T^{\mu \nu}-i A_{\mu} \star T^{\mu \nu}+i T^{\mu \nu} \star A_{\mu}=0,
$$

with

$$
T^{\mu \nu}=2\left\{F^{\mu \rho}, F_{\rho}^{\nu}\right\}_{\star}-\eta^{\mu \nu} F^{\alpha \beta} \star F_{\alpha \beta} .
$$

$T^{\mu \nu}$ is symmetric and expressed in terms of field strength only. But due to the peculiarities of gauge theory on non-commutative spaces, it is not gauge invariant. Under a gauge transformation $\delta A_{\mu}=D_{\mu} \Omega$, it transforms covariantly as

$$
T^{\mu \nu} \rightarrow \Omega \star T^{\mu \nu} \star \Omega^{-1} .
$$

Eq. (27) implies that $\partial_{\mu} \operatorname{tr} T^{\mu \nu}$ is equal to a $\star$ commutator, which gives no contribution after integration over space-time. In addition, the integrated $\operatorname{tr} T^{\mu \nu}$ is gauge invariant. Therefore one could use the integral over $\operatorname{tr} T^{\mu \nu}$ to define global gauge invariant conserved quantities 0 .

Let us be more ambitious and look for a locally conserved and gauge invariant energy-momentum tensor. For all local operators transforming covariantly under gauge transformations, one can construct gauge invariant Fourier modes by attaching a suitably adapted Wilson functional [20, 21]. Transforming back to ordinary space one then arrives at local gauge invariant quantities [24]. For our $T^{\mu \nu}$, this yields the gauge invariant tensor

$$
\hat{T}^{\mu \nu}(y)=\frac{1}{(2 \pi)^{D}} \int d k d x e^{i k y} e^{-i k x} \star \operatorname{tr}\left(U(k, x) \star T^{\mu \nu}(x)\right),
$$

with

$$
U(k, x)=P_{\star} \exp \left(i \int_{0}^{1} d t A_{\mu}(x+t \theta k) \theta^{\mu \nu} k_{\nu}\right) .
$$

$P_{\star}$ denotes path ordered $\star$ multiplication, from right to left with increasing contour parameter. The $\star$ multiplication refers to the functional dependence on $x$.

As a side remark, let us mention that this construction becomes more natural if one takes into account the remarkable identity [22, 23, 24]

$$
e_{\star}^{-i k \hat{x}}=e^{-i k x} \star U(k, x) .
$$

\footnotetext{
${ }^{6}$ To be more precise, this construction would require the absence of time-space noncommutativity.
} 
The subscript $\star$ on the exponential on the left hand side indicates that the multiplication in the Taylor series of its argument is $\star$. Here $\hat{x}^{\mu}=x^{\mu}+\theta^{\mu \nu} A_{\nu}$ is the gauge covariant coordinate introduced in ref. [25]. A straightforward proof of this identity can be given by showing that, as a function of the scale of $k, e^{i k x} \star e_{\star}^{-i k \hat{x}}$ and $U(k, x)$ fulfil the same first order differential equation with the same initial condition. Using (32), the construction in (30) simply corresponds to going forward and backward into momentum space while replacing in the first step $e^{-i k x}$ by $e_{\star}^{-i k \hat{x}}$.

Taking the derivative of (30) with respect to its argument $y$ and treating the arising factor $k$ under the integral as a derivative with respect to $x$ yields

$$
\partial_{\mu} \hat{T}^{\mu \nu}(y)=\frac{1}{(2 \pi)^{D}} \int d k d x e^{i k y} e^{-i k x} \star \operatorname{tr}\left(\frac{\partial}{\partial x^{\mu}} U(k, x) \star T^{\mu \nu}+U \star \partial_{\mu} T^{\mu \nu}(x)\right) .
$$

The derivative of $U(k, x)$ with respect to $x$ is related to a shift of the straight line from $x$ to $x+\theta k$ as a whole. One finds

$$
\frac{\partial}{\partial x^{\mu}} U(k, x)=i A_{\mu}(x+\theta k) \star U-i U \star A_{\mu}(x)+i P_{\star}\left(\int_{0}^{1} d s F_{\mu \alpha}(x+s \theta k) \theta^{\alpha \beta} k_{\beta} U\right) .
$$

Inserting this into (33) and substituting (27), we arrive at

$\partial_{\mu} \hat{T}^{\mu \nu}(y)=\frac{i}{(2 \pi)^{D}} \int d k d x e^{i k y} e^{-i k x} \star \operatorname{tr} P_{\star}\left(\int_{0}^{1} d s F_{\mu \alpha}(x+s \theta k) \theta^{\alpha \beta} k_{\beta} U(k, x) T^{\mu \nu}(x)\right)$.

Now the factor $k_{\beta}$ under the integral on the r.h.s. can be thought of as being generated by a differentiation with respect to $y^{\beta}$. This finally gives us the local conservation law

$$
\partial_{\mu} \mathbf{T}^{\mu \nu}=0
$$

where

$$
\begin{aligned}
\mathbf{T}^{\mu \nu}(y)= & \frac{1}{(2 \pi)^{D}} \int d k d x e^{i k y} e^{-i k x} \star \operatorname{tr}\left[U(k, x) \star T^{\mu \nu}(x)\right. \\
& \left.-\theta^{\mu \alpha} P_{\star}\left(\int_{0}^{1} d s F_{\alpha \beta}(x+s \theta k) U(k, x) T^{\beta \nu}(x)\right)\right] .
\end{aligned}
$$

As in the scalar case, our locally conserved $\mathbf{T}^{\mu \nu}$ is not symmetric. In four dimensions, $T$ as defined in (28) is traceless but $\mathbf{T}$ has nonvanishing trace.

\section{$4 \quad$ Summary and Outlook}

To summarize, the application of the Noether method to non-commutative field theories which admit continuous symmetries yields locally conserved currents. In 
particular, invariance of the action under space-time translations yields a locally conserved energy-momentum tensor. For the non-commutative scalar field theory with action (2), this is given by eq. (12). The fact that this tensor is not symmetric does not lead to any obvious contradictions (at least at the classical level) because the theory is not Lorentz invariant and the angular momentum density is not conserved. The fact that it is not traceless for massless fields is likewise allowed because the theory is not scale invariant. If one considers the variation of (2) under arbitrary infinitesimal diffeomorphisms under which $\theta^{\mu \nu}$ transforms like a contravariant antisymmetric tensor, then the response is governed by a non-symmetric but locally conserved tensor (see eq. (21)).

In the case of non-commutative gauge theory with gauge group $U(N)$, invariance of action (26) under infinitesimal translations and gauge transformations yields the symmetric and covariantly conserved energy-momentum tensor (28) which however is not gauge invariant. In order to construct a gauge invariant and locally conserved energy-momentum tensor, one may attach Wilson functionals in a suitable manner; this procedure yields the non-symmetric tensor (37). Finally, we note that, as emphasized by the authors of [13], their string theoretic construction of an (symmetric and locally conserved) energy-momentum tensor and a pure field theoretic study are not related in an obvious manner, since the resulting tensors correspond to different orders in an expansion in the string tension. Nevertheless, it is remarkable that in our field theoretical analysis field strength insertions into Wilson functionals also play a crucial role.

\section{Acknowledgements}

We would like to thank J. Gracia-Bondia for useful discussions and for pointing out ref. [19]. M. A. is supported by the Swiss National Science Foundation under grant number 83EU-056178. H. D. is partially supported by the Deutsche Forschungsgemeinschaft.

\section{References}

[1] T. Filk, Divergences in a Field Theory on Quantum Space, Phys. Lett. B376 (1996) 53.

[2] S. Minwalla, M. van Raamsdonk and N. Seiberg, Noncommutative Perturbative Dynamics, JHEP 0002 (2000) 020, hep-th/9912072. 
[3] M. van Raamsdonk and N. Seiberg, Comments on Noncommutative Perturbative Dynamics, JHEP 0003 (2000) 035, hep-th/0002186.

[4] R. Gopakumar, S. Minwalla and A. Strominger, Noncommutative Solitons, JHEP 0005 (2000) 020, hep-th/0003160.

[5] N. Ishibashi, S. Iso, H. Kawai and Y. Kitazawa, Wilson Loops in NonCommutative Yang-Mills, Nucl. Phys. B573 (2000) 573, hep-th/9910004.

[6] M. Abou-Zeid and H. Dorn, Dynamics of Wilson Observables in NonCommutative Gauge Theory, Phys. Lett. B504 (2001) 165, hep-th/0009231].

[7] A. Connes, M. Douglas and A. Schwarz, Noncommutative Geometry and Matrix Theory: Compactification on Tori, JHEP 9802 (1998) 003, hep-th/9711162.

[8] N. Seiberg and E. Witten, String Theory and Noncommutative Geometry, JHEP 9909 (1999) 032, hep-th/9908142.

[9] O. Andreev and H. Dorn, Diagrams of Noncommutative $\Phi^{3}$ Theory from String Theory, Nucl. Phys. B583 (2000) 145, hep-th/0003113.

[10] A. Micu and M. Sheikh-Jabbari, Noncommutative $\phi^{4}$ Theory at Two Loops, JHEP 0001 (2001) 025, hep-th/0008057.

[11] T. Pengpan and X. Xiong, Remarks on the Noncommutative Wess-Zumino Model, Phys. Rev. D63 (2001) 085012, hep-th/0009070].

[12] A. Gerhold, J. Grimstrup, H. Grosse, L. Popp, M. Schweda and R. Wulkenhaar, The Energy-Momentum Tensor on Non-Commutative Spaces - Some Pedagogical Comments, hep-th/0012112.

[13] Y. Okawa and H. Ooguri, How Noncommutative Gauge Theory Couples to Gravity, Nucl. Phys. B599 (2001) 55, hep-th/0012218].

[14] Y. Okawa and H. Ooguri, Energy-Momentum Tensors in Matrix Theory and in Non-Commutative Gauge Theories, [hep-th/0103124.

[15] H. Liu and J. Michelson, $\star$-Trek: the One Loop $N=4$ Noncommutative SYM Action, hep-th/0008205.

[16] T. Mehen and M. Wise, Generalized «-Products, Wilson Lines and the Solution of the Seiberg-Witten Equations, JHEP 0012 (2000) 008, hep-th/0010204. 
[17] C. Callan, S. Coleman and R. Jackiw, A New Improved Energy-Momentum Tensor, Ann. Phys. 59 (1970) 42.

[18] S. Coleman and R. Jackiw, Why Dilatation Generators Do Not Generate Dilatations, Ann. Phys. 67 (1971) 552.

[19] R. Jackiw and N. Manton, Symmetries and Conservation Laws in Gauge Theories, Ann. Phys. 127 (1980) 257.

[20] S. Das and S.-J. Rey, Open Wilson Lines in Noncommutative Gauge Theory and Tomography of Holographic Dual Supergravity, Nucl. Phys. B590 (2000) 453, [hep-th/0008042.

[21] D. Gross, A. Hashimoto and N. Itzhaki, Observables of Non-Commutative Gauge Theories, hep-th/0008075.

[22] A. Dhar and S. Wadia, A Note on Gauge Invariant Operators in Noncommutative Gauge Theories and the Matrix Model, Phys. Lett. B495 (2000) 413, hep-th/0008144.

[23] D. Bak, K. Lee and J. Park, Comments on Noncommutative Gauge Theories, Phys. Lett. B501 (2001) 305, hep-th/0011244.

[24] D. Berenstein and R. Leigh, Observations on Non-commutative Field Theories in Coordinate Space, hep-th/0102158.

[25] J. Madore, S. Schraml, P. Schupp and J. Wess, Gauge Theory on Noncommutative Spaces, Eur. Phys. J. C16 (2000) 161, hep-th/0001203. 